\title{
Paul Trap Simulator Experiment (PTSX) to Simulate Intense Beam Propagation Through a Periodic Focusing Quadrupole Field'
}

\author{
Ronald C. Davidson, Philip C. Efthimion, Erik Gilson, Richard Majeski \\ and Hong Qin
}

Plasma Physics Laboratory, Princeton University, Princeton, New Jersey 08543

\begin{abstract}
The Paul Trap Simulator Experiment (PTSX) is under construction at the Princeton Plasma Physics Laboratory to simulate intense beam propagation through a periodic quadrupole magnetic field. In the Paul trap configuration, a long nonneutral plasma column is confined axially by dc voltages on end cylinders at $z=+L$ and $z=-L$, and transverse confinement is provided by segmented cylindrical electrodes with applied oscillatory voltages $\pm V_{0}(t)$ over $90^{\circ}$ segments. Because the transverse focusing force is similar in waveform to that produced by a discrete set of periodic quadrupole magnets in a frame moving with the beam, the Paul trap configuration offers the possibility of simulating intense beam propagation in a compact laboratory facility. The experimental layout is described, together with the planned experiments to study beam mismatch, envelope instabilities, halo particle production, and collective wave excitations.
\end{abstract}

\section{PAUL TRAP CONFIGURATION}

Periodic focusing accelerators and transport systems have a wide range of applications ranging from basic scientific research in high energy and nuclear physics, to applications such as spallation neutron sources, tritium production, heavy ion fusion, and nuclear waste transmutation, to mention a few examples [1-5]. Of particular interest, at the high beam currents and charge densities of practical interest, are the combined effects of the applied focusing field and the intense self fields produced by the beam space charge and current on determining detailed equilibrium, stability, and transport properties. The purpose of this paper is to describe the Paul Trap Simulator Experiment (PTSX) under construction at the Princeton Plasma Physics Laboratory that fully simulates the collective processes and nonlinear transverse dynamics of an intense charged particle beam propagating through a periodic quadrupole magnetic field configuration. The idea of using a single-species trap to model periodically-focused beam propagation has been discussed by Davidson, et al. [6], and by Okamoto and Tanaka [7], although the emphasis of their work is on solenoidal confinement [7], whereas the present paper focuses on periodic quadrupole confinement [1-6]. The equivalence of the Paul trap configuration to intense beam propagation through a periodic focusing quadrupole field is discussed in Ref. 6, and in Secs. 2 and 3 of this paper we describe the experimental configuration,

${ }^{1}$ Research supported by the Department of Energy and in part by the Office of Naval Research. 


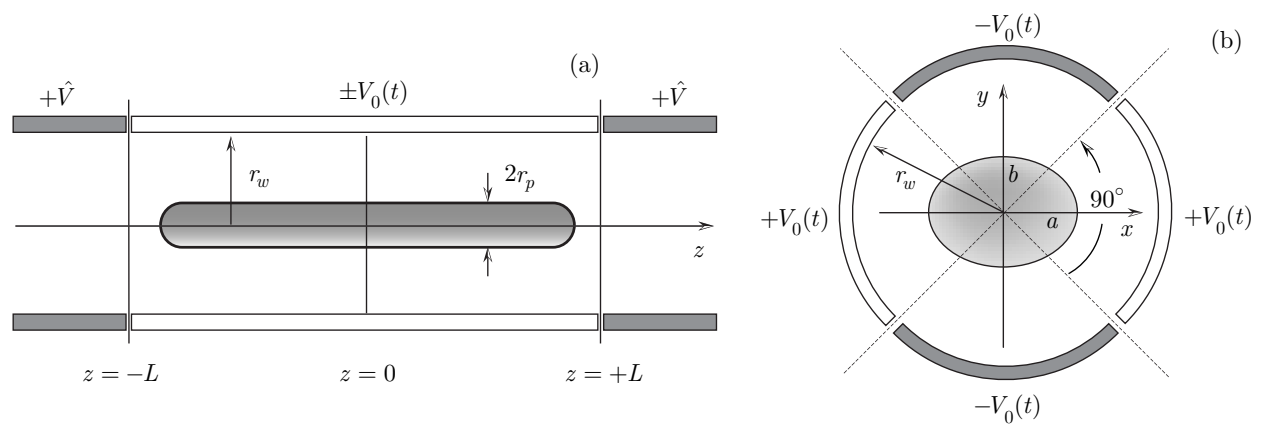

FIGURE 1. (a) Side view and (b) end view of Paul trap configuration.

and the planned experimental studies, respectively.

As illustrated in Fig. 1, to model an axially continuous charged particle beam (or a very long charge bunch), we consider a long one-component plasma column with length $2 L$ and characteristic radius $r_{p} \ll L$, confined axially by applied dc voltage $\hat{V}=$ const. on end cylinders at $z= \pm L$. The particles making up the nonrelativistic nonneutral plasma in Fig. 1 have charge $q$ and mass $m$. Transverse confinement of the particles in the $x-y$ plane is provided by segmented cylindrical electrodes (at radius $r_{w}$ ) which have applied oscillatory voltages $\pm V_{0}(t)$ with $V_{0}(t+T)=V_{0}(t)$ and $\int_{0}^{T} d t V_{0}(t)=0$, where $T=$ const . is the period, and $f_{0}=1 / T$ is the oscillation frequency. It is readily shown that the applied potential $\phi_{a}(x, y, t)$ near the axis $\left(r \ll r_{w}\right)$ in Fig. 1 is given by [6]

$$
q \phi_{a}(x, y, t)=\frac{1}{2} m \kappa_{q}(t)\left(x^{2}-y^{2}\right),
$$

where $\kappa_{q}(t)$ is defined by

$$
\kappa_{q}(t) \equiv \frac{8 q V_{0}(t)}{m \pi r_{w}^{2}} .
$$

Note that $\kappa_{q}(t+T)=\kappa_{q}(t)$ and $\int_{0}^{T} d t \kappa_{q}(t)=0$, and that $\kappa_{q}(t)$ has dimensions of $(\text { time })^{-2}$. Moreover, the leading-order correction to Eq. (1) is of order $(1 / 3)\left(r / r_{w}\right)^{4}$ [6].

The transverse Hamiltonian and the corresponding nonlinear Vlasov-Poisson equations, valid for the Paul trap configuraiton in Fig. 1, are identical in form to the corresponding equations describing the trasnverse dynamics of an intense nonneutral beam propagating through a periodic quadrupole magnetic field [6]. Because the transverse focusing force is similar in waveform to that produced by a discrete set of periodic quadrupole magnets in a frame moving with the beam, the Paul trap configuration offers the possibility of simulating intense beam propagation in a compact experimental facility. The Paul Trap Simulator Experiment (PTSX) is intended to simulate continuous beam propagation in a periodic focusing transport line. 


\section{PAUL TRAP SIMULATOR EXPERIMENT}

The applied oscillatory voltage $V_{0}(t)$ typically results in a nonneutral plasma column that has a pulsating elliptical cross-section in the $x-y$ plane [6]. It is convenient to denote the on-axis $(r=0)$ plasma density by $\hat{n}$ and the corresponding plasma frequency by $\hat{\omega}_{p} \equiv\left(4 \pi \hat{n} q^{2} / m\right)^{1 / 2}$. From Eq. (2), we denote the characteristic angular oscillation frequency $\hat{\omega}_{q}$ for the transverse motion of a single particle in the (maximum) focusing field by $\hat{\omega}_{q} \equiv\left|\hat{\kappa}_{q}\right|^{1 / 2}=\left|8 q \hat{V}_{0} / \pi m r_{w}^{2}\right|^{1 / 2}$, where $\hat{V}_{0}=\left|V_{0}(t)\right|_{\text {max }}$ is the maximum applied voltage. Transverse confinement [6] of the nonneutral plasma by the oscillatory voltage requires $\hat{\omega}_{p} / \sqrt{2}<\hat{\omega}_{q}$. On the other hand, to avoid the so-called envelope instability [3] associated with an overly strong focusing field, the oscillation frequency $f_{0}$ should be several times larger than $\hat{\omega}_{q} / 2 \pi$. Combining these inequalities gives $\hat{\omega}_{p} / \sqrt{2}<\hat{\omega}_{q} \ll$ $2 \pi f_{0}$, or equivalently, for a singly-ionized pure ion plasma (ion mass number $=\mathrm{A}$ ), such as barium or lithium,

$$
1.49 \times 10^{2}\left(\frac{\hat{n}}{A}\right)^{1 / 2}<2.5 \times 10^{5}\left(\frac{\hat{V}_{0}}{A r_{w}^{2}}\right)^{1 / 2} \ll f_{0} .
$$

Here, $\hat{n}, \hat{V}_{0}, r_{w}$, and $f_{0}$ are expressed in units of $\mathrm{cm}^{-3}$, volts, cm, and $s^{-1}$, respectively. As illustrative parameters for a barium ion plasma, we take $\hat{V}_{0}=400 \mathrm{~V}$ and $r_{w}=10 \mathrm{~cm}$. Equation (3) then gives the requirements that $\hat{n}<1.1 \times 10^{7} \mathrm{~cm}^{-3}$ and that $f_{0}$ exceed many tens of $\mathrm{kHz}$.

An elevation view of the Paul Trap Simulator Experiment (PTSX) is illustrated in Fig. 2. The apparatus is aproximately $3 \mathrm{~m}$ in overall length, and consists of a 10" O.D. electropolished stainless steel chamber with metal-sealed flanges to allow bakeout to $150^{\circ} \mathrm{C}$. A centrally-located, six-way cross accomodates the laser-induced fluorescence (LIF) diagnosic. Both radial (through the six-way cross) and paraxial (through windows at the axial ends of the device) illumination of the ion population by the excitation laser are possible. The vacuum system consists of a 1,000 1/sec turbomolecular pump backed up by an oil-free roughing pump.

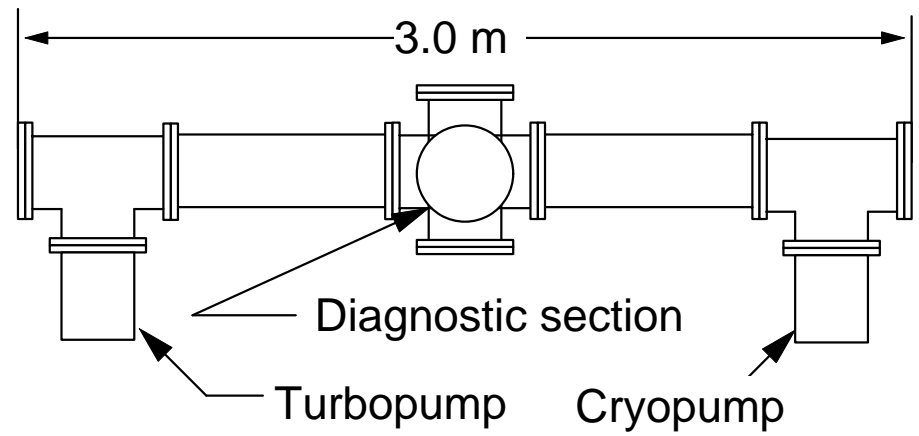

FIGURE 2. Elevation view of the Paul Trap Simulator Experiment.

The central confinement section of PTSX consists of four $200 \mathrm{~cm}$ long, azimuthally segmented stainless steel electrodes which produce the oscillatory quadrupole fields. The diameter of the confining electrode array is $20 \mathrm{~cm}$. In the central section of the trap, 
the gap between the electrodes is locally enlarged to allow passage of the laser beam for the laser-induced fluorescence diagnostic. A section of blackened high-transparency metallic mesh forms the electrodes in this section of the device, to allow imaging of the ion emission without disturbing the electric field configuration. An amplifier powers the electrodes in opposing pairs by means of a step-up transformer with two secondary windings. The amplifier is driven by a programmable waveform generator in order to permit adjustment of the waveform amplitude, pulse shape, and period in time.

Initial experiments in PTSX will utilize a barium-coated platinum or rhenium filament as an ion source, with rhenium being the favored material for good contact ionization efficiency. In this case, the spiral-wound filament is located at the midpoint of the segmented end-electrode section. In addition, a high-transparency grid is located in front of the filament, and a reflecting electrode is located behind the filament. This system is expected to be adequate for the simulation of low-intensity ion beams. If higher ion fluxes are required than can be supplied by the filament, then a barium-loaded rhenium plate will be substituted for the filament assembly.

\section{EXPERIMENTAL RESEARCH PROGRAM}

The PTSX experimental facility and associated diagnostic suite constitute a very flexible facility for simulating a wide range of nonlinear collective processes important in the propagation of intense charged particle beams over large distances. Most importantly, the flexible source geometry and ability to vary the voltage waveform $V_{0}(t)$, either dynamically or from experiment to experiment, permit detailed studies to be carried out over a wide range of density $\hat{n}$, average radius $r_{p}$, and choice of equivalent waveform for the quadrupole focusing field. Furthermore, the laser-induced fluorescence diagnostic capability will permit a detailed investigation of the evolution of the density profile, rms column radius, transverse emittance, and particle distribution function. An important dimensionless parameter $s[6]$ that measures the beam intensity and the self-field intensity is defined by $s \equiv \hat{\omega}_{p}^{2} / 2 \hat{\omega}_{q}^{2}$. Here, $\hat{\omega}_{p}$ is the plasma frequency, and $\hat{\omega}_{q}$ is the effective transverse focusing frequency due to the applied quadrupole focusing field. In the moderate-intensity beams encountered in high energy and nuclear physics applications, the self-field parameter $s$ typically satisfies $s \leq 0.2$, corresponding to emittancedominated beams which are a few (or several) Debye lengths in diameter. On the other hand, for space-charge dominated beams of interest for heavy ion fusion, the self-field parameter $s$ can approach unity, corresponding to very low transverse emittance, and near balance of the applied focusing force and the repulsive space-charge force. The PTSX facility has the capability to investigate collective nonlinear processes for values of the self-field parameter ranging from moderate self-field intensity to very high intensity.

The PTSX experimental program will focus on a variety of investigations of collective nonlinear processes of fundamental interest in the physics of intense charged particle beams. These studies will include the following areas of research.

(a) The effects of different waveforms for $V_{0}(t)$ will be determined, at fixed amplitude and frequency, on equivalent beam propagation through periodic quadrupole field geom- 
etry. Waveforms ranging from sinusoidal to a (near) step-function lattice with variable fill factor will be used. Particularly important will be a determination of the conditions for quiescent (stable) matched-beam propagation over large (equivalent) distances.

(b) At fixed values of frequency $f_{0}=1 / T$, if the focusing field amplitude is overly strong, with $\hat{\omega}_{q} T \geq \pi / 2$, it is expected that the plasma column will be subject to the so-called envelope instability [3] in which the beam envelope and rms radius develop large-amplitude oscillations. This can lead to loss of equilibrium, beam mismatch, and ejection of so-called 'halo' particles from the core. Using a sinusoidal waveform for $V_{0}(t)$, experiments will be carried out intentionially exciting the envelope instability in order to determine its detailed nonlinear evolution and saturation, dependence on the intensity parameter $s$, and its effect on the production of halo particles.

(c) A critical problem in accelerator physics is the (unwanted) production of halo particles by beam mismatch induced, for example, by faulty magnet sets which have the incorrect field intensity, or are misaligned, etc. To test this effect in the PTSX facility, we will intentionally 'spoil' the voltage waveform $V_{0}(t)$ for short times (a few or several periods) in order to induce a mismatch.

(d) In related experiments, at fixed frequency $f_{0}=1 / T$ and specified waveform (sinusoidal, say), we will adiabatically vary the amplitude of the oscillatory voltage pulse to simulate beam propagation through (long) transition regions where the focusing field amplitude is slowly varying (e.g., increasing slowly to compress the transverse dimension of the beam). Important will be a quantitative determination of how slow (i.e., over how many lattice periods) the transition has to be to assure quiescent (stable) beam propagation.

(e) One-component nonneutral plasmas and intense charged particle beams support a wide variety of collective oscillations that depend on the details of the distribution function, the focusing field strength, the self-field intensity parameter $s$, and geometric effects such as the proximity of the conducting wall. These oscillations typically involve various combinations of the frequencies $\hat{\omega}_{q}, \hat{\omega}_{p}$, and $\left(\hat{\omega}_{q}^{2}-\hat{\omega}_{p}^{2} / 2\right)^{1 / 2}$, modified by geometric effects $\left(r_{p} / r_{w}\right)$. We will carry out a series of experiments on the PTSX that investigate detailed properties of collective excitations (either excited externally, or occurring naturally as the result of beam mismatch, imperfections in the voltage waveform, or as a consequence of an instability). In addition to testing and validating theoretical models of collective oscillations in intense charged particle beams, emphasis will be placed on identifying collective modes whose signature serves as a robust diagnostic for key properties of the beam, such as line density, transverse emittance, etc.

(f) Depending on the ion source used in the PTSX experiment, there is considerable flexibility to alter the input density profiles, ranging from normal (radially decreasing or uniform profiles), to slightly hollow, to hollow, to multiple beamlets (using 'masking' techniques). It is planned to investigate a variety of physics phenomena related to the input profiles from the ion source. 


\section{CONCLUSIONS}

This paper has described the Paul Trap Simulator Experiment (PTSX), a flexible research facility to simulate intense beam propagation in periodic quadrupole field configurations. Experiments on PTSX will examine a wide range of collective processes important for intense beam propagation in alternating-gradient quadrupole field configurations.

\section{REFERENCES}

1. R. C. Davidson and H. Qin, Physics of Intense Charged Particle Beams in High Energy Accelerators (World Scientific, Singapore, 2001), and references therein.

2. A. W. Chao, Physics of Collective Beam Instabilities in High Energy Accelerators (John Wiley and Sons, Inc., New York, 1993).

3. M. Reiser, Theory and Design of Charged Particle Beams (John Wiley \& Sons, Inc., New York, 1994).

4. See, for example, Proceedings of the 1999 Particle Accelerator Conference (IEEE Catalog Number 99CH36366), pp. 1-3778.

5. See, for example, Proceedings of the International Heavy Ion Fusion Symposium, Nuclear Instruments and Methods in Physics Research A464, pp. 1-674 (2001).

6. R. C. Davidson, H. Qin, and G. Shvets, Phys. Plasmas 7, 1020 (2000).

7. H. Okamoto and H. Tanaka, Nuclear Instruments and Methods in Physics Research A437, 178 (1999).

8. W. Paul and H. Steinwedel, Z. Naturforschung A8, 448 (1953).

9. D. J. Wineland, W. M. Itano, and R. S. Vandyck, Jr., Adv. Atom. Mol. Phys. 19, 135 (1983). 